\title{
Midline2 is overexpressed and a prognostic indicator in human breast cancer and promotes breast cancer cell proliferation in vitro and in vivo
}

Lan Wang ${ }^{1}$, Jueheng $\mathrm{Wu}^{3}$, Jie Yuan ${ }^{3}$, Xun $\mathrm{Zhu}^{2,3}$, Hongmei Wu${ }^{1}$, Mengfeng Li $(\varangle)^{2,3}$

${ }^{I}$ Department of Microbiology and Immunology, School of Basic Courses, Guangdong Pharmaceutical University, Guangzhou 510006, China; ${ }^{2}$ Department of Microbiology, Zhongshan School of Medicine, Sun Yat-sen University, Guangzhou 510080, China; ${ }^{3}$ Key Laboratory of Tropical Disease Control (Sun Yat-sen University), Ministry of Education, Guangzhou 510080, China

(C) Higher Education Press 2021

Erratum to Frontiers of Medicine 2016 10(1): 41-51

https://doi.org/10.1007/s11684-016-0429-z

The original version of this article unfortunately contained some mistakes.

The immunohistochemistry (IHC) staining of MID2 in "ANT" from Patient 2 and "T" from Patient 6 in original Fig. 2C (Page 45) should be corrected. The authors apologize for this error and state that this does not change the scientific conclusions of the article in any way. The corrected figures are as following:
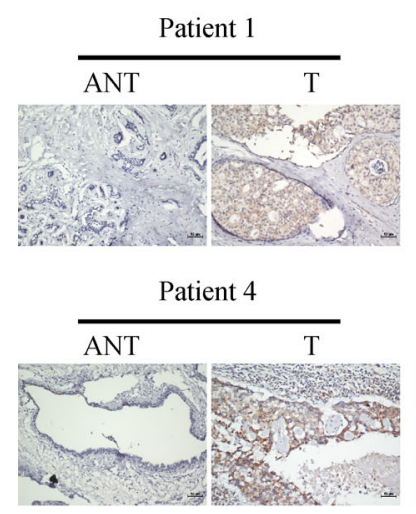
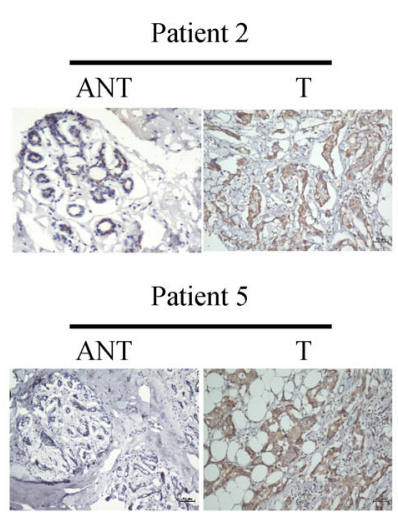
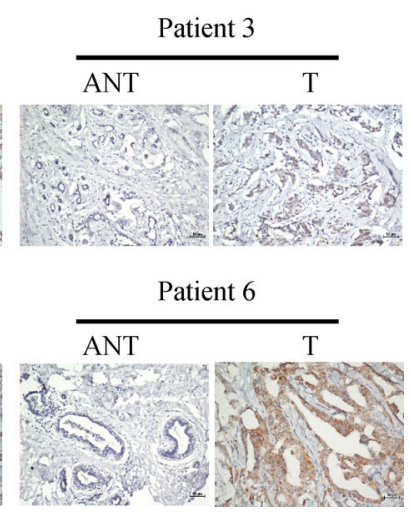

The online version of the original article can be found at https://doi.org/10.1007/s11684-016-0429-z

Correspondence: Mengfeng Li, limf@mail.sysu.edu.cn 